\title{
The Carpathian lingonberry, raspberry and blackberry fruit extracts feature variable antimicrobial efficiency strength
}

\author{
${ }^{1,2}$ Emőke Mihok - ${ }^{2}$ Éva György - ${ }^{1}$ Endre Máthé \\ ${ }^{1}$ University of Debrecen, Faculty of Agricultural and Food Sciences and Environmental Management, Institute of Food Technology, H-4032, \\ Debrecen, Böszörményi str. 128, Hungary \\ ${ }^{2}$ Sapientia Hungarian University of Transylvania, Faculty of Economics, Socio-Human Sciences and Engineering, Department of Food \\ Science, RO-530104 Miercurea Ciuc, Libertății sq. 1. Romania \\ mihok.emoke@agr.unideb.hu
}

SUMMARY

Wild berry is an excellent source of phytonutrients and/or bioactive compounds associated with significant therapeutic properties, so that they have been utilized in folk medicine and traditional nutrition throughout centuries. Multiple health-promoting effects, such as anti-inflammatory, anti-diabetic, anti-heart and coronary disease properties were attributed to such wild berries. It has also been proved that berries could feature antimicrobial effects that could be of a great importance for the prevention of food-feed poisoning and fighting back antibiotic resistance. In this study, we investigated the antimicrobial properties of lingonberry (Vaccinium vitis-idaea), raspberry (Rubus idaeus) and blackberry (Rubus fruticosus) crude and ethanolic extracts prepared from fruits obtained from the spontaneous flora of Eastern Carpathian Mountains situated in Transylvania. The antimicrobial effect of crude and alcoholic extracts were assessed on four Gram-negative, five Gram-positive bacteria and one yeast species using the agar diffusion method. The studied bacteria can cause food or feed spoilage and foodborne diseases. Our results indicate the significant inhibitory effect of lingonberry extracts in the case of Gram-negative bacteria like Proteus vulgaris and Salmonella Hartford, while among Gram-positive bacteria the strongest inhibitory effect was observed for Bacillus species like B. cereus, B. subtilis, B. mojavensis and Micrococcus luteus. The raspberry and blackberry extracts featured milder inhibitory effects in the case of the studied bacteria species. Furthermore, we have studied the crude or ethanolic extract combinations associated antimicrobial effects synergistic/additive or antagonistic properties. Interestingly, the triple and double ethanolic extract mixes had stronger antimicrobial properties, whereas the crude extract mixes showed relatively reduced effects, if any. Our results indicate that the antimicrobial activity of studied fruit extracts obtained from wild berries can vary upon the applied extraction method and their combination formulae, so that all these considerations must be taken into account when such fruit extracts are considered for foodstuff development.

Keywords: berry fruits, antimicrobial effect, juice, ethanol extract, synergism

\section{INTRODUCTION}

The nutritional and physiological importance of wild and cultivated berries is a well-known fact. The low calorie and fat content together with favorable macro- and micro-nutrient content of berries make them highly suitable for human and animal nutrition. Concomitantly the berries with antimicrobial effects could be a good alternative to traditional antibiotic therapy of humans and animals, because of their availability, a wider health-promoting spectra and palatability (Krstić et al., 2014). However, the berries are also rich in phytonutrients or bioactive substances, which could further increase the efficiency of vital phenomena in case of consumers (Cicero and Colletti, 2016). Berries contain a large amount of polyphenolic compounds featuring significant antioxidant properties (Szajdek and Borowska, 2008), though such parameters of different species of berries are highly variable.

The lingonberry (Vaccinium vitis-idaea Linné) is a wild berry fruit specific to the Northern Hemisphere temperate zone, and is considered to provide significant health benefits through its bioactive constituents. It was observed that the lingonberry has outstanding storage properties that were put on the expense of acidic phytonutrient content including benzoic acid. However, Viljakainen et al. (2002) was suggesting that the Northern Hemisphere temperate zone growing wild berries besides benzoic acid could feature highly variable malic and citric acids concentrations. As an example they have shown that the benzoic acid content of lingonberry juice varied at $0.1-0.7 \mathrm{gL}^{-1}$, a fairly large concentration range. Visti et al. (2003) have found the lingonberries having higher concentration of benzoic acid (up $1.3 \mathrm{gL}^{-1}$ of free benzoic acid) associated with $\mathrm{pH}$ 2.6-2.9 value. The benzoic acid content and storability were associated with the antimicrobial properties to the berries. Warth (1988) proposed that the concentration of $0.2-0.3 \mathrm{gL}^{-1}$ benzoic acid is sufficient to prevent the growth of Saccharomyces cerevisiae. The antimicrobial effect of wild lingonberry has been extensively studied (Lehtonen et al., 2013), and the inhibition of several bacteria like Staphylococcus aureus (Kylli et al., 2011; Paudel et al., 2014), Bacillus cereus and Staphylococcus epidermidis (Nohynek et al., 2006), Bacillus subtilis and Micrococcus luteus (Rauha et al., 2000) were demonstrated.

Raspberry (Rubus idaeus) is another wild berry species specific to the Northern Hemisphere temperate zone with human and veterinary health claims. The raspberry specific phytonutrients include a number of polar and non-polar compounds. Among the polar constituents the most relevant ones are the vitamin $\mathrm{C}$, organic acids (citric acid, malic acid and fumaric acid), sugars, volatile aromatic substances, phenolic compounds, phenolic acid (ellagic acid and derivatives, tannins), flavonoids and anthocyanins (Kafkas et al., 
2008; González et al., 2003; Krstić et al., 2014). As far as for the non-polar compounds, raspberries contain a significant amount of essential fatty acids (Parry et al., 2005; Kafkas et al., 2008). The antimicrobial effect of raspberry was put on the expense of ellagitannins acting as possible anti-adherence compounds in preventing the colonization and infection of many pathogens (Heinonen, 2007). Puupponen-Pimiä et al. (2005) showed the raspberry extract damaging the Salmonella membrane. Krisch et al. (2009) were suggesting that the antimicrobial effect of raspberry extract can be attributed to the phenolic and organic acids, inducing the acidification the cytoplasm of Gram-negative microorganisms, and causing the bacterial outer membrane disintegration. Krauze-Baranowska et al. (2014) have found the raspberry extract most effective in case of Corynebacterium diphtheriae and Moraxella catarrhalis bacteria.

Some publications on the chemical composition and physiological (anti-tumor and antidiabetic) effects of the wild blackberry (Rubus fruticosus L.) have been published (Li et al., 2015; Verma et al., 2014). All five types of blackberries produced in Mediterranean region were shown to feature very low levels of ascorbic acid and malic acid, but significant amounts of fructose were measured, while citric acid was not detected (Kafkas et al., 2006). On the other hand, the blackberries grown in Europe and North America temperate regions showed significant amounts of reduced ascorbic acid (12.4 $13.1 \mathrm{mg} 100 \mathrm{~g}^{-1}$ fresh weight), (Benvenuti et al., 2004). The phenolic compounds from blackberries are considered the most relevant phytonutrients that could be related to the prevention and/or treatment of many human conditions, including cancer, cardiovascular diseases, cataracts, diabetes, asthma, hepatitis, arthritis, immune deficiency diseases (Skrovankova et al., 2015). It has also been demonstrated that blackberry may affect the activity of several human pathogenic Listeria monocytogenes, Salmonella Typhimurium and the Escherichia coli O157:H7 bacteria (Yang et al., 2014). On the other hand, the antimicrobial effects of the blackberry have been shown in case of Gramnegative Escherichia coli, Salmonella enterica subsp. enterica, and Gram-positive Staphylococcus aureus and Bacillus cereus bacteria and Candida albicans yeast (Demirbas et al., 2017).

The main purpose of the current study was to characterize the antimicrobial properties of extracts of lingonberry, raspberry and blackberry wildly grown in the Carpathian region of Transylvania. Moreover, we set to analyze the putative synergistic and/or antagonistic antimicrobial effects of our lingonberry, raspberry and blackberry extracts.

\section{MATERIALS AND METHODS}

\section{Place and date of sampling}

Several samples corresponding to different types of berries were collected from July to October 2018, in the Carpathian region of Transylvania (Romania). The lingonberry (Vaccinium vitis-idaea) and raspberries (Rubus idaeus) were harvested from a forest area of
Harghita County, and the blackberries (Rubus fruticosus) were from the County of Mureș. After collection all the samples were stored frozen and stored at $-20{ }^{\circ} \mathrm{C}$ until further testing and extract preparation.

\section{Sample preparation}

A raw fruit juice and an ethanol extract were prepared from lingonberries (Vaccinium vitis-idaea), raspberries (Rubus idaeus) and blackberries (Rubus fruticosus). To extract the raw juice, $100 \mathrm{~g}$ of the frozen fruit was allowed to thaw, and the juice of the fruits was extracted by pressing.

During the preparation of the ethanol extract, $100 \mathrm{~g}$ of the fruit was allowed to thaw, squeezed and mixed with $300 \mathrm{ml}$ of $80 \%$ ethanol (Sigma Aldrich). The extraction went on for two hours using a magnetic stirrer at room temperature. The resulting solution was filtered, and the solvent was removed from the extract using a rotary evaporator (Heidolph Laborota 4000). The obtained berry fruit extracts were stored at $-18^{\circ} \mathrm{C}$ prior the antimicrobial assays.

In order to prepare the triple and double mixes equal amounts of individual crude and ethanolic extracts were combined, respectively.

\section{Antibacterial activity}

To detect the antimicrobial activity of the extracts, nine strains of Gram-positive and Gram-negative bacteria and one yeast species were selected, all being microorganisms that can cause food or feed deterioration, or different diseases. These selected microorganisms were: Escherichia coli, Staphylococcus aureus, Bacillus cereus, Bacillus subtilis, Bacillus mojavensis, Salmonella Hartford, Proteus vulgaris, Pseudomonas baetica, Micrococcus luteus and Saccharomyces cerevisiae. The antimicrobial effect was determined using the agar diffusion method. The microbial cultures were grown for 48 hours at $37{ }^{\circ} \mathrm{C}$ on Nutrient agar (VWR: BDH Chemicals) medium. For each bacterium and yeast species, $0.1 \mathrm{ml}$ of microbial suspension with 1.0 turbidity in physiological solution was spread with surface streaking on Nutrient agar (peptone from meat $10 \mathrm{~g}$, meat extract $10 \mathrm{~g}, \mathrm{NaCl} 5 \mathrm{~g}$, agar $18 \mathrm{~g}$, distilled water $1000 \mathrm{ml}$ ). In the center of all inoculated mediums was cut in sterile conditions a hole with $8 \mathrm{~mm}$ diameter, and in the hole was pipetted $0.1 \mathrm{ml}$ extract. Following incubation, the diameter of zones of inhibition was determined (after 48 hours), and after 96 hours the bactericidal activity was again inspected.

For the accurate evaluation of the results obtained, the average of four parallel experiments specific measurements was calculated.

The synergistic antibacterial effect was analyzed by combining equal amounts (one in one ratio) of the obtained berry extracts (lingonberry, raspberry and blackberry), and applying the agar diffusion test as described above for the following bacterial strains: Escherichia coli, Staphylococcus aureus, Bacillus cereus, B. subtilis, B. mojavensis, Salmonella Hartford, Proteus vulgaris, Pseudomonas baetica and Micrococcus luteus. 


\section{Statistical analysis}

Calculation of averages, standard deviations (SD), for the correlation between the berry extracts and the antimicrobial effects, t-tests analysis were performed using the SPSS v.22.0 (SPSS 2013) software.

\section{RESULTS AND DISCUSSION}

Our experiments are meant to shed light on the antimicrobial effects of some wild plants like lingonberry (Vaccinium vitis-idaea), raspberries (Rubus idaeus) and blackberries (Rubus fruticosus) from the spontaneous flora of the Eastern Carpathian Mountains situated in Transylvania. Assessing the antimicrobial effects of different plants species is gaining more and more relevance as the antibiotic resistance becoming more alarming phenomenon among humans and domesticated animals (for review see Pisoschi et al., 2018). Therefore, any study that would bring new insights into reducing microbiological infestations is welcomed in particular if naturally occurring solutions/extracts/compounds are assessed. It is also our aim to pay attention to the so-called regional effect so that freshly collected fruits of local plant species are analyzed, and we will continue studying samples from the same collection locations in the following years to come. Such a concept was also followed by Laslo and Köbölkuti (2017), who could show that their lingonberry extracts inhibited efficiently the growth of Gram-negative Pseudomonas fluorescens and Pseudomonas aeruginosa bacteria. Moreover, they were also stating that the antibacterial effect of lingonberry crude extract depends on its own bioactive compound profile that could be influenced by environmental conditions, harvesting period in conjugation with ripening and the storage methods.

We set ourselves to carry out a comparative study in order to analyze the antimicrobial effect of lingonberry, raspberry and blackberry, while for each fruit species two type of extracts (crudes, ethanolic) were prepared, and tested using the agar diffusion method as described in Materials and Methods. The obtained results for Gram-negative bacteria and Grampositive bacteria - yeast are shown separately in Tables 1 and 2 .

Table 1

Antimicrobial effect of berry fruit extracts on Gram-negative bacteria (list the name of bacteria) $n=4, \pm S D$

\begin{tabular}{|c|c|c|c|c|c|c|}
\hline \multirow{3}{*}{$\begin{array}{c}\text { Extract samples } \\
\text { Bacteria }\end{array}$} & \multicolumn{3}{|c|}{ Crude extract } & \multicolumn{3}{|c|}{ Ethanolic extract } \\
\hline & Lingonberry & Raspberry & Blackberry & Lingonberry & Raspberry & Blackberry \\
\hline & \multicolumn{6}{|c|}{ Inhibition zone diameter (mm) } \\
\hline Escherichia coli & $11.28 \pm 3.36$ & $11.09 \pm 1.47$ & $9.37 \pm 1.25$ & $19.48 \pm 2.64$ & $12.31 \pm 0.39$ & $16.70 \pm 2.36$ \\
\hline Proteus vulgaris & $13.21 \pm 2.15$ & $15.05 \pm 1.26$ & $12.75 \pm 1.49$ & $21.62 \pm 1.96$ & $21.54 \pm 2.45$ & $20.53 \pm 1.29$ \\
\hline Salmonella Hartford & $13.74 \pm 3.24$ & $13.42 \pm 2.62$ & $14.49 \pm 1.53$ & $20.96 \pm 2.61$ & $13.70 \pm 0.67$ & $9.54 \pm 1.58$ \\
\hline Pseudomonas baetica & $10.40 \pm 1.08$ & $11.83 \pm 1.28$ & $9.76 \pm 2.68$ & $15.82 \pm 3.43$ & $17.79 \pm 0.54$ & $14.30 \pm 0.90$ \\
\hline
\end{tabular}

As shown in Table 1 , the crude extracts of lingonberry, raspberry and blackberry featured similar inhibitory effects for all tested Gram-negative bacteria species. There were no significant differences between the observed results regarding the inhibitory effect $(\mathrm{P}>0.05)$. Interestingly the ethanol extracts of lingonberry, raspberry and blackberry proved to be more competitive in inhibiting the growth of Gramnegative bacteria as compared to the corresponding crude extracts. The $E$. coli growth was suppressed most efficiently by the lingonberry ethanol extract $(19.48 \pm 2.64 \mathrm{~mm})$, while in case of Proteus vulgaris both lingonberry $(21.62 \pm 1.96 \mathrm{~mm})$ and raspberry $(21.54 \pm 2.45 \mathrm{~mm})$ ethanolic extracts were effective, though the blackberry specific effect was also relatively high $(20.53 \pm 1.29 \mathrm{~mm})$. The lingonberry ethanolic extract was undoubtedly the most competitive in impeding the growth of Salmonella Hartford, while the raspberry ethanolic extract was the most effective in hindering the growth of Pseudomonas baetica. Taken together, our experiments are indicating a more efficient Gram-negative antibacterial effect for the lingonberry ethanolic extract that is closely followed by the raspberry ethanolic extract. It is also worth mentioning that the Proteus vulgaris species proved to be the most sensitive to the individual lingonberry, raspberry and blackberry ethanolic extracts. Taken together, our observations regarding the crude extracts inhibitory effects on Gram-negative bacteria, we could observe that none of the studied crude extracts showed an exceedingly high value, while for the ethanolic extracts, the recorded diameter specific values of inhibition zone were slightly higher but of the same order of magnitude.

Assessing the Gram-positive specific antimicrobial effect of crude and ethanolic extracts of lingonberry, raspberry and blackberry revealed efficacy differences with the ethanolic extracts being more competitive as compared to the crude extracts (for details see Table 2). 
Antimicrobial effect of berry fruit extracts on Gram-positive bacteria and yeast (list the name of bacteria) $\mathbf{n}=6$, \pm SD

\begin{tabular}{|c|c|c|c|c|c|c|}
\hline \multirow{3}{*}{$\begin{array}{c}\text { Extract samples } \\
\text { Bacteria and yeast }\end{array}$} & \multicolumn{3}{|c|}{ Crude extract } & \multicolumn{3}{|c|}{ Ethanolic extract } \\
\hline & Lingonberry & Raspberry & Blackberry & Lingonberry & Raspberry & Blackberry \\
\hline & \multicolumn{6}{|c|}{ Inhibition zone diameter $(\mathrm{mm})$} \\
\hline Bacillus cereus & $14.79 \pm 0.56$ & $12.59 \pm 1.26$ & $11.20 \pm 2.37$ & $23.54 \pm 0.86$ & $18.54 \pm 1.80$ & $14.00 \pm 3.86$ \\
\hline Bacillus subtilis & $8.50 \pm 1.33$ & $7.57 \pm 1.21$ & $8.10 \pm 1.97$ & $23.36 \pm 2.22$ & $15.69 \pm 2.16$ & $14.04 \pm 4.67$ \\
\hline Bacillus mojavensis & $12.82 \pm 0.94$ & $13.07 \pm 1.59$ & $9.79 \pm 0.83$ & $23.18 \pm 4.23$ & $18.08 \pm 2.96$ & $15.43 \pm 2.07$ \\
\hline Micrococcus luteus & $16.69 \pm 4.31$ & $11.87 \pm 0.85$ & $10.64 \pm 1.43$ & $28.08 \pm 5.65$ & $16.77 \pm 2.18$ & $15.00 \pm 1.92$ \\
\hline Staphylococcus aureus & $14.39 \pm 1.37$ & $10.00 \pm 0.78$ & $7.28 \pm 4.86$ & $19.56 \pm 5.30$ & $15.14 \pm 2.63$ & $15.64 \pm 1.11$ \\
\hline Saccharomyces cerevisiae & $10.40 \pm 0.78$ & ND & ND & $15.82 \pm 3.39$ & ND & $11.52 \pm 0.99$ \\
\hline
\end{tabular}

ND: not detectable

Concerning the berry-specific Gram-positive antimicrobial effect concerns, the lingonberry crude extract proved to be the most competitive in the case of Bacillus cereus, Bacillus subtilis, Micrococcus luteus, Staphylococcus aureus and Saccharomyces cerevisiae. The raspberry crude extract seemed to have the strongest inhibitory effect $(13.07 \pm 1.59 \mathrm{~mm})$ on Bacillus mojavensis, while the suppressive effect of the lingonberry crude extract had almost the same efficiency $(12.82 \pm 0.94 \mathrm{~mm})$. The blackberry crude extracts antimicrobial effects were lagging behind the lingonberry and raspberry extracts as suggested by the sizes of inhibition zone diameters. Among the ethanolic extracts once again the lingonberry came across like the most competitive inhibitor of the Gram-positive bacteria, while the raspberry and blackberry ethanolic extracts were less efficient. The growth of Saccharomyces cerevisiae was only inhibited by both the crude and ethanolic extracts of lingonberry. The ethanolic extract $(15.82 \pm 3.39 \mathrm{~mm})$ of lingonberry featured a stronger inhibition than the crude extract $(10.40 \pm 0.78 \mathrm{~mm})$ as inferred by the sizes of inhibition zones. However, with the exception of ethanolic blackberry extract, the crude blackberry and both the crude and ethanolic extracts of raspberry exerted no detectable suppressive influence on the growth of Saccharomyces cerevisiae. Summarizing the antimicrobial effects of the studied extracts on the Gram-positive bacteria and yeast it seems obvious that the ethanolic extracts were more efficient than the crude extracts, and once again the observed values were of the same magnitude for both type of extracts.

Having seen the individual antimicrobial effects of both crude and ethanolic extracts of lingonberry, raspberry and blackberry, we carry out a complementarity study by combining together either the crude or the ethanolic extracts. To test the efficacy of every extract combination, the diameter of inhibition zone was determined upon the use of diffusion method, and the obtained results are shown in Table 3.

The antimicrobial effect of the combination of crude and ethanol extract mixes (list the name of bacteria) $\mathrm{n}=9, \pm \mathrm{SD}$

\begin{tabular}{|c|c|c|c|c|c|c|c|c|}
\hline \multirow{2}{*}{ Extract samples } & \multicolumn{4}{|c|}{ Crude extract } & \multicolumn{4}{|c|}{ Ethanolic extract } \\
\hline & LRB & LR & $\mathrm{RB}$ & LB & LRB & LR & $\mathrm{RB}$ & LB \\
\hline Bacteria & \multicolumn{8}{|c|}{ Inhibition zone diameter (mm) } \\
\hline \multicolumn{9}{|l|}{ Gram-negative } \\
\hline Escherichia coli & $11.70 \pm 2.25$ & ND & ND & ND & $19.05 \pm 3.47$ & $24.71 \pm 1.55$ & $20.70 \pm 4.87$ & $22.14 \pm 0.89$ \\
\hline Proteus vulgaris & $14.26 \pm 1.48$ & $12.39 \pm 1.89$ & $11.01 \pm 0.83$ & $14.06 \pm 0.39$ & $24.20 \pm 3.33$ & $20.50 \pm 2.28$ & $23.88 \pm 4.84$ & $22.92 \pm 4.56$ \\
\hline $\begin{array}{l}\text { Salmonella } \\
\text { Hartford }\end{array}$ & $14.26 \pm 1.48$ & $12.17 \pm 0.67$ & $10.96 \pm 0.34$ & $10.24 \pm 0.33$ & $18.42 \pm 0.96$ & $17.87 \pm 0.83$ & $14.32 \pm 1.68$ & $22.61 \pm 4.44$ \\
\hline $\begin{array}{l}\text { Pseudomonas } \\
\text { baetica }\end{array}$ & ND & $10.92 \pm 1.88$ & ND & $12.74 \pm 2.06$ & $19.62 \pm 1.03$ & $22.08 \pm 2.26$ & $17.88 \pm 2.14$ & $23.68 \pm 4.64$ \\
\hline \multicolumn{9}{|l|}{ Gram-positive } \\
\hline Bacillus cereus & $14.08 \pm 1.61$ & $14.64 \pm 2.85$ & $11.60 \pm 1.65$ & $12.11 \pm 1.07$ & $21.61 \pm 1.45$ & $21.19 \pm 0.37$ & $19.42 \pm 2.27$ & $26.60 \pm 2.82$ \\
\hline Bacillus subtilis & $13.42 \pm 2.03$ & $12.27 \pm 1.69$ & $11.10 \pm 1.00$ & $12.92 \pm 0.89$ & $21.05 \pm 2.31$ & $23.44 \pm 2.28$ & $17.44 \pm 2.10$ & $20.58 \pm 2.76$ \\
\hline Bacillus mojavensis & $17.43 \pm 2.03$ & $16.63 \pm 3.46$ & $13.61 \pm 3.60$ & $13.29 \pm 3.10$ & $26.46 \pm 5.27$ & $27.00 \pm 3.16$ & $24.18 \pm 3.96$ & $28.94 \pm 1.81$ \\
\hline Micrococcus luteus & $12.25 \pm 3.16$ & $10.84 \pm 1.32$ & $11.06 \pm 1.55$ & $12.46 \pm 2.93$ & $31.08 \pm 1.04$ & $31.45 \pm 2.77$ & $26.49 \pm 1.11$ & $32.65 \pm 1.11$ \\
\hline $\begin{array}{l}\text { Staphylococcus } \\
\text { aureus }\end{array}$ & $12.21 \pm 0.84$ & $10.59 \pm 0.58$ & $9.43 \pm 0.21$ & $8.98 \pm 0.23$ & $21.64 \pm 1.23$ & $23.93 \pm 0.75$ & $15.51 \pm 1.07$ & $20.00 \pm 1.57$ \\
\hline
\end{tabular}

LRB: lingonberry-raspberry-blackberry extract, LR: lingonberry-raspberry extract, RB: raspberry-blackberry extract, LB: lingonberryblackberry extract, ND: not detectable

In the case of E.coli, the triple lingonberryraspberry-blackberry (LRB) crude extract combination and the lingonberry-raspberry (LR) double ethanolic extract showed the most efficient antibacterial effect.
Surprisingly for lingonberry-raspberry (LR), raspberryblackberry (RB) and lingonberry-blackberry (LB) double crude extracts we could not detect any inhibition zone, suggesting that such mixed extracts do not inhibit 
at any extent the growth of the examined E.coli culture. Among the alcoholic mixed extracts, the LR double combination showed the most efficient suppressive effect in the case of E.coli that was followed by the LB double combination. The Proteus vulgaris was the most efficiently inhibited by the LRB triple and LB double crude extract and the LRB triple ethanolic extract combinations. The Salmonella Hartford growth was the most seriously affected by the LRB triple crude extract and the LB double ethanolic extract combinations. The Pseudomonas baetica culture was the most efficiently set back by the LR or LB double crude extracts and the LB double ethanolic extract combinations. It is worth mentioning that the LRB triple and the RB double crude extracts did not show any inhibition with respect to the growth of Pseudomonas baetica. The Bacillus cereus was most efficiently set back by the LR crude and LB double ethanolic extract mixes. The Bacillus subtilis inhibition was most effective in the case of LRB crude and ethanolic LR and LRB extract mixes. The most potent antibacterial effect on Bacillus mojavensis was featured by the crude LRB and LR extracts, while on the site of the ethanolic extract mixes the LB proved the most effective. In the case of Micrococcus luteus, the crude and ethanolic LB extracts acted most intensively, with latest being the strongest antimicrobial effect detected throughout our experiments. Finally, for the Staphylococcus aureus, the strongest suppressor effect was observed applying the crude LRB and ethanolic LR extracts.

The analysis of the antibacterial effects of extract mixes revealed greater inhibitory values than in the cases of individual extracts, but these data were again of the same magnitude, so they could indicate the existence of some putative additive or synergistic effects. In our concept the additive interactions are seen as the sum of the effects of individual extracts, while synergistic interactions would represent a situation when the effects of the extract mixes is greater than the sum of their constituent extracts taken independently from each other and at the same doses. Based on our experimental setup, it is rather inadequate to compare the individual extracts data with the triple or double extract mixes specific data since the concentrations of fruit extracts are different. Accordingly, the triple extract mixes contain one third, while the double extract mixes contain only half of the constituents seen in the case of single extracts. Nevertheless, in situations like the ethanolic LR double extract treated E.coli, or the ethanolic LRB triple extract mix treated Proteus vulgaris, or the ethanolic LB double extract treated Salmonella Hartford, or the ethanolic LB double extract treated Micrococcus luteus, the obtained inhibitory effect related data exceeds those seen in the case of individual extracts, so that we can predict the existence of some synergetic interactions. It remains an open question to assess the concentration dependency of such presumptive synergistic interactions.

Interestingly, the absence of inhibitory effect for $E$. coli in the case of crude LR, RB and LB extract mixes could be an indicative of some antagonistic interaction between the given extracts (see Table 3 ). We can also presume a similar situation in the case Pseudomonas baetica where the crude LRB and RB extract mixes had no detectable inhibitory effect. Envisioning the antagonism as the opposite of additive interactions would be a simplistic approach, but most probably cannot be fully applied to our experiments because contrary to the above mentioned crude LR, RB and LB double extracts (without any suppressive effect), the triple extracts do feature some inhibitory effect. This would mean that the triple LRB extract would bring together a bioactive compound profile that will gain some inhibitory competence.

\section{CONCLUSION}

Our results indicate that the lingonberry (Vaccinium vitis-idaea), raspberry (Rubus idaeus) and blackberry (Rubus fruticosus) extracts feature variable antimicrobial efficiency strength. We were also able to demonstrate that the efficiency of antimicrobial effect could be influenced by the type of the applied fruit extract (crude vs. ethanolic), suggesting that the methods used to generates the crude and ethanolic extracts may generate significant compositional differences. Compositional differences among plant extracts have already been invoked by others to explain antimicrobial effectiveness. According to PuupponenPimiä et al. (2004), the antimicrobial effects of lyophilized berries, phenolic extracts and fractions was dependent upon the phenolic content in case of the Staphylococcus bacteria. Our experiments are suggesting that the fruit made alcoholic extracts were more efficient than the crude ones among the tested microorganisms.

It is possible that, besides the compositional differences of the applied berry extracts, the studied microorganisms also feature variable sensitivity that could be put on the expense of some species specific structural and/or regulatory characteristics. Puupponen-Pimiä et al. (2001) observed that their berry extracts inhibited the growth of Gram-negative Salmonella and Escherichia strains, but were inefficient for the Gram-positive Lactobacillus species. Taken together our observations are indicating that the tested Gram-negative and Gram-positive bacteria were likely more prone to inhibition in case of lingonberry and raspberry extracts (either crude or ethanolic), while the blackberry extracts were showing slightly reduced but significant inhibitory effects. It is important to pinpoint that we were able to further demonstrate the lingonberries inhibitory effect on Saccharomyces cerevisiae, an observation previously reported by Cioch et al. (2017). Moreover, to our knowledge we are the first to report the blackberries antimicrobial effect on Saccharomyces cerevisiae.

Finally, we would like to stress that the different combination of lingonberry, raspberry and blackberry extracts based inhibitory tests on selected Gramnegative and Gram-positive bacteria panels are suggesting synergistic/additive and/or antagonistic interactions regarding the antimicrobial effect. The above mentioned interactions together with the 
chemical composition of the studied berry extracts should be further clarified in order to define novel antimicrobial applications for lingonberry, raspberry and blackberry to combat food and feed poisoning and/or foodborne diseases.

\section{ACKNOWLEDGEMENTS}

The work/publication is supported by the EFOP3.6.3-VEKOP-16-2017-00008 project. The project is co-financed by the European Union and the European Social Fund.

\section{REFERENCES}

Benvenuti, S.-Pellati, F.-Melegari, M. A.-Bertelli, D. (2004) Polyphenols, anthocyanins, ascorbic acid, and radical scavenging activity of Rubus, Ribes, and Aronia. Journal of Food Science. 69. 3. FCT164-FCT169.

Cicero, A. F.-Colletti, A. (2016): Role of phytochemicals in the management of metabolic syndrome. Phytomedicine. 23. 11. 1134-1144.

Cioch, M.-Satora, P. A. W. E. Ł.-Skotniczny, M.-Semik-Szczurak, D.-Tarko, T. (2017): Characterisation of Antimicrobia Properties of Extracts of Selected Medicinal Plants. Polish Journal of Microbiology. 66. 4. 463-472.

Demirbas, A. -Yilmaz, V.-Ildiz, N.-Baldemir, A.-Ocsoy, I. (2017) Anthocyanins-rich berry extracts directed formation of AgNPs with the investigation of their antioxidant and antimicrobial activities. Journal of Molecular Liquids. 248. 1044-1049.

González, E. M.-de Ancos, B.-Cano, M. P. (2003): Relation between bioactive compounds and free radical scavenging capacity in berry fruits during frozen storage. Journal of the Science of Food and Agriculture. 83. 7. 722-726.

Heinonen, M. (2007): Antioxidant activity and antimicrobial effect of berry phenolics - a Finnish perspective. Molecular Nutrition and Food Research. 51. 6. 684-691.

Kafkas, E.-Koşar, M.-Türemiş, N.-Başer, K. H. C. (2006): Analysis of sugars, organic acids and vitamin $\mathrm{C}$ contents of blackberry genotypes from Turkey. Food Chemistry. 97. 4. 732-736.

Kafkas, E.-Özgen, M.-Özoğul, Y.-Türemiş, N. (2008) Phytochemical and fatty acid profile of selected red raspberry cultivars: A comparative study. Journal of Food Quality. 31. 1. $67-78$.

Krauze-Baranowska, M.-Majdan, M.-Hałasa, R.-Głód, D.-Kula M.-Fecka, I.-Orzeł, A. (2014): The antimicrobial activity of fruits from some cultivar varieties of Rubus idaeus and Rubus occidentalis. Food and Function. 5. 10. 2536-2541.

Krisch, J.-Ördögh, L.-Galgóczy, L.-Papp, T.-Vágvölgyi, C. (2009): Anticandidal effect of berry juices and extracts from Ribes species. Open Life Sciences. 4. 1. 86-89.

Krstić, T. P.-Suvajdžić, L. D.-Stojanović, S. Z.-Velhner, M. J.Milanov, D. S.-Bojić, G. M.-Ilić, N. M. (2014): Different antimicrobial effects of raspberry depending on the method of active components isolation. Food and Feed Research. 41. 2. 125-130.

Kylli, P.-Nohynek, L.-Puupponen-Pimiä, R.-WesterlundWikström, B.-Leppänen, T.-Welling, J.-Moilanen E.Heinonen, M. (2011): Lingonberry (Vaccinium vitis-idaea) and european cranberry (Vaccinium microcarpon) proanthocyanidins: isolation, identification, and bioactivities Journal of Agricultural and Food Chemistry. 59. 7. 3373-3384.

Laslo, É.-Köbölkuti, Z. A. (2017): Total phenol content and antimicrobial activity of lingonberry (Vaccinium vitis-idaea L.) from several areas in the Eastern Carpathians. Notulae Scientia Biologicae. 9. 1. 77-83.

Lehtonen, H. M.-Lindstedt, A.-Järvinen, R.-Sinkkonen, J.-Graça, G.-Viitanen, M.-Gil, A. M. (2013): 1H NMR-based metabolic fingerprinting of urine metabolites after consumption of lingonberries (Vaccinium vitis-idaea) with a high-fat meal. Food chemistry. 138. 2-3. 982-990.

Li, J.-Du, L. F.-He, Y.-Yang, L.-Li, Y. Y.-Wang, Y. F.-Chai, X.Zhu, Y.-Gao, X. M. (2015): Chemical Constituents and Biological Activities of Plants from the Genus Rubus. Chemistry and Biodiversity. 12. 12. 1809-1847.
Nohynek, L. J.-Alakomi, H. L.-Kähkönen, M. P.-Heinonen, M.Helander, I. M.-Oksman-Caldentey, K. M.-Puupponen-Pimiä, R. H. (2006): Berry phenolics: antimicrobial properties and mechanisms of action against severe human pathogens. Nutrition and cancer. 54. 1. 18-32.

Parry, J.-Su, L.-Luther, M.-Zhou, K.-Yurawecz, M. P.-Whittaker, P.-Yu, L. (2005): Fatty acid composition and antioxidant properties of cold-pressed marionberry, boysenberry, red raspberry, and blueberry seed oils. Journal of Agricultural and Food Chemistry. 53. 3. 566-573.

Paudel, B.-Bhattarai, H. D.-Kim, I. C.-Lee, H.-Sofronov, R.Ivanova, L.-Poryadina, L.-Yim, J. H. (2014): Estimation of antioxidant, antimicrobial activity and brineshrimp toxicity of plants collected from Oymyakon region of the Republic of Sakha (Yakutia), Russia. Biological Research. 47. 1 10-16.

Pisoschi, A. M.-Pop, A.-Georgescu, C.-Turcuş, V.-Olah, N. K.Mathe, E. (2018): An overview of natural antimicrobials role in food.nEuropean Journal of Medicinal Chemistry. 143. 922-935.

Puupponen-Pimiä, R.-Aura, A. M.-Karppinen, S.-OksmanCaldentey, K. M.-Poutanen, K. (2004): Interactions between plant bioactive food ingredients and intestinal flora-effects on human health. Bioscience and Microflora. 23. 2. 67-80.

Puupponen-Pimiä, R.-Nohynek, L.-Alakomi, H. L.-OksmanCaldentey, K. M. (2005): Bioactive berry compounds-novel tools against human pathogens. Applied Microbiology and Biotechnology. 67.1. 8-18.

Puupponen-Pimiä, R.-Nohynek, L.-Meier, C.-Kahkonen, M.Heinonen, M.-Hopia, A.-Oksman-Caldentey, K. M. (2001): Antimicrobial properties of phenolic compounds from berries. Journal of Applied Microbiology. 90. 4. 494-507.

Rauha, J. P.-Remes, S.-Heinonen, M.-Hopia, A.-Kähkönen, M.Kujala, T.-Pihlaja, K.-Vuorela, H.-Vuorela, P. (2000): Antimicrobial effects of Finnish plant extracts containing flavonoids and other phenolic compounds. International Journal of Food Microbiology. 56. 1. 3-12.

Skrovankova, S.-Sumczynski, D.-Mlcek, J.-Jurikova, T.-Sochor, J. (2015): Bioactive compounds and antioxidant activity in different types of berries. International Journal of Molecular Sciences. 16. 10. 24673-24706.

Szajdek, A.-Borowska, E. J. (2008): Bioactive compounds and health-promoting properties of berry fruits: a review. Plant Foods for Human Nutrition. 63. 4. 147-156.

Verma, R.-Gangrade, T.-Punasiya, R.-Ghulaxe, C. (2014): Rubus fruticosus (blackberry) use as an herbal medicine. Pharmacognosy Reviews. 8. 16. 101.

Viljakainen, S.-Visti, A.-Laakso, S. (2002): Concentrations of organic acids and soluble sugars in juices from Nordic berries. Acta Agriculturae Scandinavica. Section B-Plant Soil Science. 52. 2. 101-109.

Visti, A.-Viljakainen, S.-Laakso, S. (2003): Preparation of fermentable lingonberry juice through removal of benzoic acid by Saccharomyces cerevisiae yeast. Food Research International. 36. 6. 597-602.

Warth, A. D. (1988): Effect of benzoic acid on growth yield of yeasts differing in their resistance to preservatives. Applied and Environmental Microbiology. 54. 8. 2091-2095.

Yang, H.-Hewes, D.-Salaheen, S.-Federman, C.-Biswas, D. (2014): Effects of blackberry juice on growth inhibition of foodborne pathogens and growth promotion of Lactobacillus. Food Control. 37. 15-20. 